\title{
Food for thought about manipulating gut bacteria
}

\section{Nathalie M. Delzenne \& Laure B. Bindels}

\section{Knowing how dietary fibre nourishes gut microorganisms might suggest ways to boost health-promoting bacteria. A method developed to pinpoint bacteria that consume particular types of dietary fibre could advance such efforts.}

Certain gut microorganisms can boost human health, but it is unclear how diet could be harnessed to easily manipulate the composition of gut microbes to boost the levels of desired bacteria. Writing in Cell, Patnode et al. ${ }^{1}$ present a useful approach for assessing interactions between human gut microbes and the dietary fibre that sustains their existence.

Dietary fibre is promoted as part of a healthy diet worldwide. Many people, however, do not achieve their recommended fibre intake because they consume insufficient fruit, vegetables and cereals. Inadequate fibre intake is associated with common conditions including obesity, diabetes and cancer ${ }^{2}$. Yet understanding the mechanisms that link fibre-rich food to good health is challenging. Dietary fibre encompasses a wide range of complex molecules, most of which are present in plant cells; among them are carbohydrate molecules called glycans, which are resistant to digestion by human enzymes. As a consequence, some ingested fibre is excreted unchanged in faeces, whereas most is metabolized by gut microbes.

These microbes have a diverse and extremely complex metabolic capacity. Bacteria that express different enzymes for metabolizing fibre can survive and grow using a range of foods. Some bacterial species might compete with each other for the same food source, which could lower the abundance of species that compete less successfully. How might gut microbes be manipulated through human dietary intervention? For example, the concept of using prebiotics compounds that affect gut microbes, thereby benefiting the human host - has been proposed. One such idea is to use particular fibre sources that provide food for the desired gut microbes $^{3,4}$. However, determining whether dietary fibre can promote health in this way requires a sophisticated understanding of the interactions that occur when the complex community of gut microbes encounters a source of fibre.

Previous work ${ }^{5}$ had indicated that transferring the gut microbes of human twins who have contrasting body masses (obese and lean) into mice induced a corresponding difference in the animals' body masses. However, when some of the obese mice were housed with

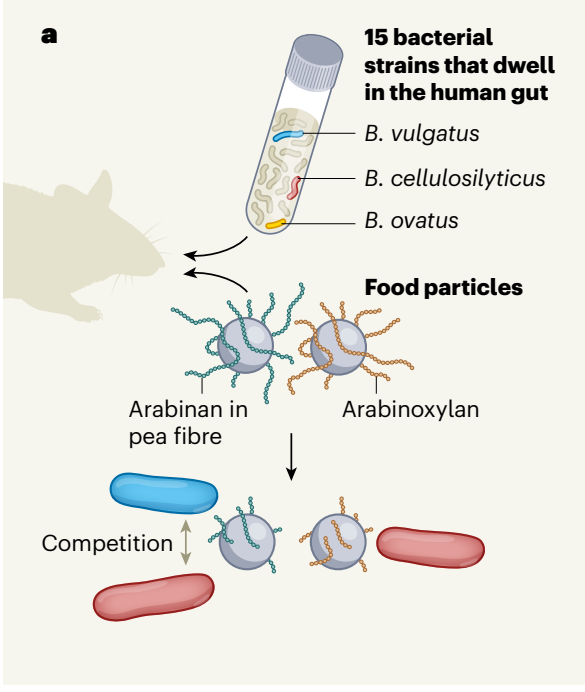

the lean mice, they had less adipose fat than did obese animals that were not co-housed with lean mice - and this weight-loss effect correlated with the transfer of Bacteroides bacterial species from the lean mice to the obese mice ${ }^{5}$. High consumption of fibre-rich plant foods was required for this adipose-fat reduction to occur ${ }^{5}$. However, the types of fibre responsible for this effect, and how these interact with specific gut microorganisms, was unknown. Patnode and colleagues now reveal how particular types of glycan can drive competition between different Bacteroides species resident in the human gut.

Patnode et al. studied mice that lacked their normal microbes, and instead harboured 15 strains of gut-dwelling bacteria from a lean human who had an obese twin. The authors fed the mice different combinations of fibre sources as part of their diet. Analysing faecal samples enabled the researchers to track how the diets affected the relative abundance of each bacterial species in the animals' gut. This approach pinpointed, for example, a dose-response effect of pea fibre on the relative abundance of Bacteroides thetaiotaomicron in the bacterial population, as well as a pronounced effect of certain

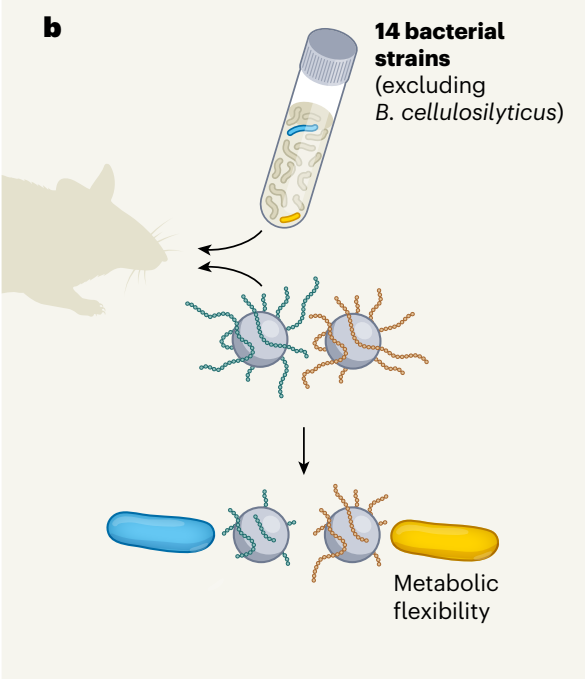

Figure 1 | Investigating how human gut-dwelling bacteria metabolize dietary fibre. a, Patnode et al. ${ }^{1}$ gave mice that lacked their natural gut microbes a set of 15 bacterial strains that dwell in the human gut, including the species Bacteroides cellulosilyticus, Bacteroides ovatus and Bacteroides vulgatus. The authors developed a method for tracking fibre digestion. They generated magnetic beads coated with a fibre of interest, and fed these beads (termed food particles) to the animals. Applying a magnetic field enabled the recovery of food particles and assessment of the extent of fibre degradation. The animals received food particles that included some coated with pea fibre that is rich in the molecule arabinan, and some coated with the molecule arabinoxylan. B. vulgatus and B. cellulosilyticus competed to degrade the arabinan, B. cellulosilyticus degraded arabinoxylan, and B. ovatus degraded other molecules (not shown). b, When the experiment was repeated without $B$. cellulosilyticus, B. ovatus demonstrated metabolic flexibility, by switching to degrade arabinoxylan. B. ovatus degraded less arabinoxylan than did B. cellulosilyticus. 


\section{News \& views}

types of barley fibre ( $\beta$-glucan and bran) on the relative abundance of Bacteroides ovatus. These results reveal the specificity of the effects that different forms of dietary fibre can have on bacterial populations.

To identify the genes required for a specific bacterium of interest to metabolize fibre, the authors gave mice bacterial strains that were engineered to contain mutations at random sites across their genome, and fed the animals different kinds of dietary fibre. By analysing the proteins in mouse faecal samples, the authors identified a set of bacterial proteins that allow certain microbes to grow successfully in particular feeding regimes. For example, when mice received dietary fibre from fruit peelings (citrus pectin) that are rich in a type of molecule called methylated homogalacturonan, this led to a rise in the expression of proteins that degrade such molecules in the bacterium Bacteroides cellulosilyticus. And when mice received pea fibre, which is rich in a polymer molecule called arabinan (which contains the sugar arabinose), the expression of proteins involved in arabinan degradation rose in the bacterium $B$. thetaiotaomicron.

Perhaps the most original part of this research is the development of artificial 'food particles' consisting of glycan-coated magnetic beads (Fig. 1) that can be administered orally to mice and recovered by applying a magnetic field. Patnode et al. used this strategy to investigate how bacterial species respond to different food sources by assessing the extent of glycan degradation in the recovered beads. When mice that had been colonized only with B. cellulosilyticus or Bacteroides vulgatus were given food particles coated with pea fibre, the levels of arabinose in the recovered beads were lower than the original levels, demonstrating that both of these bacterial species had metabolized this molecule in vivo.

In a parallel experiment, mice were colonized either with all 15 bacterial strains from the lean twin, or with 14 of the strains (B. cellulosilyticus excluded), before being given food particles containing pea fibre (Fig. 1). The level of degradation of arabinose in the arabinan-rich pea-fibre beads was then compared, and was found to be the same in both cases. This suggests that some change occurs in the bacterial community, in the absence of $B$. cellulosilyticus, that enables arabinose from pea fibre to be degraded as much as it would be if all 15 bacterial strains were present. The story might be different for other forms of dietary fibre.

Along with the food particles coated with pea fibre, the animals received some coated with molecules of arabinoxylan (a polymer of the sugars arabinose and xylose). However, in the case of arabinoxylan, the bacterial strains were less able to process this molecule when B. cellulosilyticus was absent than when it was present, and the arabinoxylan-metabolizing activity was attributed to B. ovatus. In the absence of B. cellulosilyticus, $B$. ovatus undergoes a metabolic shift that boosts its ability to use arabinoxylan. When both $B$. ovatus and $B$. cellulosilyticus were absent from the bacterial populations, arabinoxylan-coated beads retained their original levels of arabinose, revealing that none of the remaining 13 bacterial strains took advantage of arabinoxylan availability.

This study reveals the flexibility and adaptability of gut microbes in response to their nutritional environment. It provides a useful focus on specific forms of dietary fibre and bacterial species known to be linked to diet-associated resistance to a rise in adipose tissue $^{5}$. This 'simplification' of the context suggests a way forward in understanding the key genes and proteins of Bacteroides that are crucial for the degradation of dietary fibre, and that might affect the abundance of particular gut bacteria. The findings also reveal how $B$. cellulosilyticus can have a dominant role in its interactions with certain bacteria with which it can compete for the same food source. The work also uncovers hidden metabolic flexibility, such as the ability of
B. ovatus to adapt its metabolic strategy.

When assessing this study, it is worth bearing in mind that Bacteroides is not the only type of bacterium that commonly uses dietary fibre for food, and that the fibre-containing foods tested by the authors are not the major sources of dietary fibre in a typical human diet. Moreover, the abundance of Bacteroides varies enormously between people ${ }^{6}$, and the hypothesis that key Bacteroides species might affect the success of dieting efforts to control obesity requires further investigation.

Although it concentrates on Bacteroides only, Patnode and colleagues' work represents useful progress towards developing personalized nutrition strategies for tailoring gut microbes in the future. The study also complements other research ${ }^{7-9}$ that explores how bacteria in the human gut might contribute to the body's response to a particular diet. Thanks to Patnode et al., we have fresh insights into how specific types of bacterium use and compete for dietary fibre. Future research will undoubtedly continue to refine the link between fibre-rich food and health, by taking into account the role of the gut microbial community.

Nathalie M. Delzenne and Laure B. Bindels are at the Louvain Drug Research Institute, Metabolism and Nutrition Research Group, Catholic University of Louvain, 1200 Brussels, Belgium.

e-mail: nathalie.delzenne@uclouvain.be

1. Patnode, M. L. et al. Cell 179, 59-73 (2019).

2. Delzenne, N. M. et al. Clin. Nutr. https://doi.org/10.1016/ j.clnu.2019.03.002 (2019).

3. Bindels, L. B., Delzenne, N. M., Cani, P. D. \& Walter, J. Nature Rev. Gastroenterol. Hepatol. 12, 303-310 (2015)

4. Gibson, G. R. et al. Nature Rev. Gastroenterol. Hepatol. 14, 491-502 (2017).

5. Ridaura, V. K. et al. Science 341, 1241214 (2013).

6. The Human Microbiome Project Consortium. Nature 486, 207-214 (2012).

7. Salonen, A. et al. ISME J. 8, 2218-2230 (2014).

8. Bindels, L. B. et al. Microbiome 5, 12 (2017).

9. Zhao, L. et al. Science 359, 1151-1156 (2018). 